\title{
Estimation of Diffusion Properties in Crossing Fiber Bundles
}

\author{
Matthan W. A. Caan*, H. Ganesh Khedoe, Dirk H. J. Poot, Arjan J. den Dekker, Silvia D. Olabarriaga, \\ Kees A. Grimbergen, Lucas J. van Vliet, and Frans M. Vos
}

\begin{abstract}
There is an ongoing debate on how to model diffusivity in fiber crossings. We propose an optimization framework for the selection of a dual tensor model and the set of diffusion weighting parameters $b$, such that both the diffusion shape and orientation parameters can be precisely as well as accurately estimated. For that, we have adopted the Cramér-Rao lower bound (CRLB) on the variance of the model parameters, and performed Monte Carlo simulations. We have found that the axial diffusion $\lambda_{\|}$ needs to be constrained, while an isotropic fraction can be modeled by a single parameter $f_{\text {iso }}$. Under these circumstances, the Fractional Anisotropy (FA) of both tensors can theoretically be independently estimated with a precision of $9 \%($ at SNR $=25)$. Levenberg-Marquardt optimization of the Maximum Likelihood function with a Rician noise model approached this precision while the bias was insignificant. A two-element $b$-vector $b=\left[\begin{array}{ll}1.0 & 3.5\end{array}\right]$ • $10^{3} \mathrm{~mm}^{-2} \mathrm{~s}$ was found to be sufficient for estimating parameters of heterogeneous tissue with low error. This has allowed us to estimate consistent FA-profiles along crossing tracts. This work defines fundamental limits for comparative studies to correctly analyze crossing white matter structures.
\end{abstract}

Index Terms-Cramér-Rao analysis, diffusion properties, diffusion weighted magnetic resonance imaging, dual tensor model, Monte Carlo simulations.

Manuscript received March 30, 2010; revised April 27, 2010; accepted April 27, 2010. Date of publication June 17, 2010; date of current version August 04, 2010. This work was supported by the BiG Grid project for the use of the computing and storage facilities, with financial support from the Nederlandse Organisatie voor Wetenschappelijk Onderzoek (Netherlands Organisation for Scientific Research, NWO). Asterisk indicates corresponding author.

*M. W. A. Caan is with the Delft University of Technology, Imaging Science and Technology, 2628 CJ Delft, The Netherlands, and also with the Academic Medical Center Amsterdam, Radiology, 1105 AZ Amsterdam, The Netherlands (e-mail: m.w.a.caan@tudelft.nl).

H. G. Khedoe is with the Delft University of Technology, Imaging Science and Technology, 2628 CJ Delft, The Netherlands (e-mail: h.g.khedoe@tudelft. $\mathrm{nl})$.

D. H. J. Poot is with the Physics Department, University of Antwerp, 2610 Antwerp, Belgium (e-mail: dirk.poot@ua.ac.be).

A. J. den Dekker is with the Delft University of Technology, Delft Center for Systems and Control, 2628 CD Delft, The Netherlands (e-mail: a.j.dendekker@tudelft.nl).

S. D. Olabarriaga and C. A. Grimbergen are with the University of Amsterdam, Academic Medical Center, Amsterdam, 1105 AZ The Netherlands (e-mail: s.d.olabarriaga@amc.uva.nl; c.a.grimbergen@amc.uva.nl).

L. J. van Vliet is with the Delft University of Technology, Imaging Science and Technology, 2628 CJ Delft, The Netherlands (e-mail: 1.j.vanvliet@tudelft. $\mathrm{nl})$.

F. M. Vos is with the Delft University of Technology, Imaging Science and Technology, 2628 CJ Delft, The Netherlands, and also with the Academic Medical Center Amsterdam, Radiology, 1105 AZ Amsterdam, The Netherlands (e-mail: f.m.vos@tudelft.nl).

Color versions of one or more of the figures in this paper are available online at http://ieeexplore.ieee.org.

Digital Object Identifier 10.1109/TMI.2010.2049577

\section{INTRODUCTION}

$\mathbf{T}$ HE integrity of white matter structures in the brain is frequently studied by means of diffusion Weighted magnetic resonance imaging (DW-MRI). Classically, the diffusion in the brain is described by a rank-two diffusion tensor which is estimated from the diffusion weighted images (DWIs). The local geometry of axons is quantified by measures such as the fractional anisotropy (FA), which measures the anisotropy of the diffusion process within a voxel. The orientation information, extracted from the DWIs, allows the reconstruction of white matter bundles by means of fiber tractography [1]

There is an ongoing debate on how to model diffusivity in fiber crossings. Here the diffusion profile cannot be adequately described by a single tensor [2]. We propose an optimization framework for the estimation of diffusion parameters at such locations. Our focus is on unbiased and highly reproducible estimation of both the diffusion shapes and the orientations of the fiber bundles. The diffusion shapes reveal fiber integrity that can be used as biomarkers: both in animal [3] and human brain tissue [4], axial and radial diffusivities were related to underlying biology. Concurrently, the optimal acquisition parameters $\mathbf{b}$ are determined to facilitate the diffusion parameter estimation. It has already been noted that when diffusivity information in multiple compartments is required, data need to be acquired at multiple b-values [5]. We assume constant scanning time and uniformly distributed gradient directions. We will use the framework to determine the optimal dual tensor model. It will be shown that the axial diffusion of the two tensors involved must be constrained (taken identical), while an isotropic fraction can be simultaneously modeled by a single parameter. Moreover, it will be demonstrated that under these circumstances the FA of the both tensors can be independently estimated with high precision.

We adopt the Cramér-Rao lower bound (CRLB) on the variance of the dual tensor model parameters to determine in theory the optimal precision of the parameters. Monte Carlo simulations are performed to study the degree to which this precision is achieved in practice and to address a potential bias in the estimation. We will verify the findings by reconstructing FA-profiles along crossing fiber bundles.

The relevance of our work is in comparative studies where the goal is to assess subtle differences in diffusion shape between patients and matched controls. As an example, an increase in FA in the posterior limb of the internal capsule, as reported in a study of schizophrenia [6], might need reinterpretation due to possibly incorrect single tensor modeling in the presence of 
the crossing superior longitudinal fasciculus. A study of Amyotrophic Lateral Sclerosis reported decreased FA-values in the corticospinal tract, except at the crossing with the corpus callosum [7]. Preliminary work on estimation of diffusion shape parameters of a dual tensor model resulted in unstable results [8].

\section{BACKGROUND}

\section{A. Diffusion Modeling}

We aim to quantify the independent diffusion profiles of two axonal bundles that appear as "crossing fibers" within the typical resolution of DW-MRI (2 $\mathrm{mm}$ at a field strength of 3.0T). A number of solutions has been proposed for these situations, mainly focusing on reconstructing fiber orientations. Two- or multi-tensor approaches [9]-[11], higher order tensors [12] or constrained spherical deconvolution [13] parametrically extend conventional diffusion tensor imaging. Nonparametric approaches have also emerged, including $q$-ball imaging [14]. Tractography results are becoming more accurate when performed in a dual tensor field [15]-[17] or in a fiber orientation distribution field [18].

Assuming the diffusion to be mono-exponential and Gaussian, the measured diffusion weighted signal $S_{j}$ is initially modeled to contain a contribution of up to two fiber bundles and an isotropic part

$$
S_{j}=S_{0}\left\{\sum_{i=1,2} f_{i} \exp \left(-b_{j} \mathbf{g}_{\mathbf{j}}^{\mathbf{T}} \mathbf{D}_{\mathbf{i}} \mathbf{g}_{\mathbf{j}}\right)+f_{\text {iso }} \exp \left(-b_{j} D_{\text {iso }}\right)\right\}
$$

with $S_{0}$ being the signal without diffusion weighting, $b_{j}$ the amount of diffusion weighting corresponding to a gradient direction $\mathbf{g}_{\mathbf{j}}$, selected from a vector $\mathbf{b}$ of length $n_{\mathbf{b}}$ with unique components, $\mathbf{D}_{\mathbf{1}}$ and $\mathbf{D}_{\mathbf{2}}$ the $(3 \times 3)$ diffusion tensors of the two fibers, $D_{\text {iso }}$ the scalar amount of isotropic diffusion and $f_{i}$ the volume fractions of the diffusion processes summing up to one, i.e., $f_{1}+f_{2}+f_{\text {iso }}=1$.

An incorrect representation of the noise properties, particularly assuming a Gaussian instead of a Rician noise distribution in the DWIs, may also render an inappropriate (i.e., biased) signal model [19]. A single tensor model was extended by estimating the Rician noise level in a maximum likelihood framework [20], [21]. Rician noise reduction by spatial regularization [22], [23] can be used to limit the bias in estimates of a single tensor model. Recently, the linear minimum mean square error (LMMSE) estimator [24] was proposed to decrease the noise in DWIs in an efficient way.

Previously, the Fisher Information matrix was used to assess the expected (co)variance of parameters of a single tensor model [20], which was also explored by means of an error propagation framework [25].

\section{B. Optimization of Diffusion Imaging}

The diffusion weighting parameter $b$ selects the diffusivity scale of the measurements. The amount of diffusion weighting is determined by the diffusion weighting parameter $b$, which is calculated from the selected gradient strength and diffusion time
$\Delta-(1 / 3) \delta$, where $\Delta$ is the time between both gradients and $\delta$ the diffusion weighting gradient duration [26].

The expected diffusivity values determine the range for which $b$ is optimal. Measurements of the apparent diffusion coefficients (ADC) in white matter show values in the range of 0.25 . $10^{-3}$ to $1.5 \cdot 10^{-3} \mathrm{~mm}^{2} \mathrm{~s}^{-1}$ [27]. At first glance the inverse ${ }^{1}$ diffusion values indicate that $b$-values in the range of $b=0.7$. $10^{3} \mathrm{~mm}^{-2} \mathrm{~s}$ to $2.5 \cdot 10^{3} \mathrm{~mm}^{-2} \mathrm{~s}$ should be selected. For lower $b$-values, only isotropic diffusion between axonal boundaries is measured. Measuring at higher $b$-values $\left(b>3.0 \cdot 10^{3} \mathrm{~mm}^{-2} \mathrm{~s}\right)$ will introduce a sensitivity to different compartments such as the myelin sheet [30], giving rise to restricted and hindered diffusion [31]. In the latter case, the Gaussian diffusion assumption is no longer valid, which is beyond the scope of this work.

For a broad range of applications (such as stroke), these diffusivity values are best determined with a $b$-value of $1.0 \cdot 10^{3} \mathrm{~mm}^{-2} \mathrm{~s}[32]$, by means of a spin-echo EPI sequence with typically 6-45 gradient directions [7]. Multiple fiber orientations are best modeled at a higher diffusion weighting contrast using $b=2.0 \cdot 10^{3} \mathrm{~mm}^{-2} \mathrm{~s}$ [8], [33]. Multiexponential signal decay emanating from multicompartments in a study to Multiple Sclerosis was only visible at an even higher $b$-value of $3.0 \cdot 10^{3} \mathrm{~mm}^{-2} \mathrm{~s}$ [34]. In order to independently estimate two diffusion profiles, a vector $\mathbf{b}$ containing two distinct values $\left(n_{\mathbf{b}}=2\right)$ must be used [5].

The DW-signal is increasingly attenuated with higher $b$-values, as is shown in (1), such that its signal-to-noise ratio $\left(\mathrm{SNR}_{\mathrm{DW}}\right)$ is optimal at low $b$-values $\left(b<0.5 \cdot 10^{3} \mathrm{~mm}^{-2} \mathrm{~s}\right.$ [35]). For high values $\left(b>3.0 \cdot 10^{3} \mathrm{~mm}^{-2} \mathrm{~s}\right)$ noise dominates the signal for all expected diffusion values. Concurrently, a low diffusion weighting implies poor contrast between low and high diffusivities. The contrast-to-noise ratio is thus expected to be optimal for intermediate $b$-values.

By minimizing the CRLB, $\mathbf{b}$ was optimized for higher ratios of the maximum and minimum diffusion coefficients in the sample, modeled by a single tensor [36]. Maximal gradient strengths for varying axon diameters were found in a composite hindered and restricted model of diffusion, allowing for measuring direct tissue-microstructure features [37].

\section{THEORY}

\section{A. Dual Tensor Model Parameterization}

The dual tensor model [(1)] needs to be parameterized such that its estimated values reside in a well-defined range. We will parameterize $\mathbf{D}_{\mathbf{i}}$ by its eigenvalues $\mathbf{E}_{\mathbf{i}}$ and rotations $\mathbf{R}_{\mathbf{i}}, \mathbf{D}_{\mathbf{i}}=$ $\mathbf{R}_{\mathbf{i}}^{\mathbf{T}} \mathbf{E}_{\mathbf{i}} \mathbf{R}_{\mathbf{i}}$. The matrices $\mathbf{R}_{\mathbf{i}=\mathbf{1 , 2}}$ describe rotations around the $x-, y$ - and $z$-axes: $\mathbf{R}_{\mathbf{i}}\left(\alpha_{1-4}\right)=\mathbf{R}_{\mathbf{x}}\left(\alpha_{1}\right) \mathbf{R}_{\mathbf{y}}\left(\alpha_{2}\right) \mathbf{R}_{\mathbf{z}}\left(\alpha_{3} \pm \alpha_{4}\right)$. The first two rotations $\mathbf{R}_{\mathbf{x}}\left(\alpha_{1}\right) \mathbf{R}_{\mathbf{y}}\left(\alpha_{2}\right)$ determine the orientation of the plane in which the principal eigenvectors of both tensors reside; $\mathbf{R}_{\mathbf{z}}\left(\alpha_{3} \pm \alpha_{4}\right)$ represents the mean orientation $\alpha_{3}$ with deviations $\pm \alpha_{4}$ of the principal eigenvectors of the two tensors $\mathbf{i}=1,2$. The eigenvalues are contained in the diagonal matrices $\mathbf{E}_{\mathbf{i}}$. The diffusion perpendicular to the fiber orientation is assumed to be isotropic such that its eigenvalues are identical [5], which reduces the number of free parameters. Let $\lambda_{i \|}$ and

${ }^{1}$ The naive inverse balances reported optimal values for $b$ of $0.85 / D[28]$ and $1.1 / D[29]$ 
TABLE I

Dual Tensor Models With Increasing Degrees of Freedom. Parameters May be Estimated ('+'), Constrained to Other Parameters (E.G., $\lambda_{2 \|}=\lambda_{1 \|}$ ), Set Constant, OR OMitTed ('0'). IN EACH Model the Noise LeVEl $\sigma$ IS Set to the Value Estimated With Model \#2. the Units of the Diffusion Values are $\{\lambda, D\} \cdot 10^{-3} \mathrm{~mm}^{2} \mathrm{~s}^{-1}$. Mean $\overline{\boldsymbol{\theta}}$ and Standard Deviation of the Parameter Values std $(\boldsymbol{\theta})$ USED IN THE EXPERIMENTS, IN WHICH THE ANGLES $\alpha_{1-3}$ ARE RANDOMLY CHOSEN (RND)

\begin{tabular}{|c|c|c|c|c|c|c|c|c|c|c|}
\hline model & $\lambda_{1 \|}$ & $\lambda_{1 \perp}$ & $\lambda_{2 \|}$ & $\lambda_{2 \perp}$ & $\alpha_{1-3}$ & $\alpha_{4}$ & $f_{1}$ & $f_{\text {iso }}$ & $D_{\text {iso }}$ & $\sigma$ \\
\hline \hline$\# 1$ & + & + & + & + & + & + & + & 0 & 0 & $\sigma_{\# 2}$ \\
\hline$\# 2$ & + & + & $\lambda_{1 \|}$ & + & + & + & + & 0 & 0 & + \\
\hline$\# 3$ & + & + & $\lambda_{1 \|}$ & + & + & + & + & + & 3.0 & $\sigma_{\# 2}$ \\
\hline$\# 4$ & + & + & $\lambda_{1 \|}$ & + & + & + & + & + & + & $\sigma_{\# 2}$ \\
\hline$\overline{\boldsymbol{\theta}}$ & 1.4 & 0.4 & 1.4 & 0.3 & rnd & $\frac{\pi}{5}$ & 0.4 & 0.15 & 3.0 & \\
\hline $\operatorname{std}(\boldsymbol{\theta})$ & 0.2 & 0.07 & 0.2 & 0.07 & rnd & $0.1 \cdot \frac{\pi}{5}$ & 0.05 & 0.03 & 0 & \\
\hline
\end{tabular}

$\lambda_{i \perp}$ denote respectively the axial and perpendicular diffusion. Now $\mathbf{E}_{\mathbf{i}}$ yields

$$
\mathbf{E}_{\mathbf{i}}=\operatorname{diag}\left(\begin{array}{lll}
\lambda_{i \|} & \lambda_{i \perp} & \lambda_{i \perp}
\end{array}\right), i \in\{1,2\} .
$$

Non-negativity of diffusivity values is imposed by adopting the exponential mappings $\exp (\cdot)$. The model needs to be further constrained such that the eigenvalues are sorted, i.e., $\lambda_{i \|}>\lambda_{i \perp}$. For that purpose, the axial diffusivity $\lambda_{i \|}$ is computed by adding the average perpendicular diffusivity $(1 / 2)\left(\lambda_{1 \perp}+\lambda_{2 \perp}\right)$ to a positive difference between axial and perpendicular diffusivity $\exp \Delta \tilde{\lambda}_{i \|}$. This formulation also allows us to impose an equality constraint on $\lambda_{1 \|}$ and $\lambda_{2 \|}$ (see Table I below). Volume fractions are defined in the range $0<f_{i}<1$ by error functions $\operatorname{erf}(\cdot)$, in which $\operatorname{erf}(x)=(2 / \sqrt{\pi}) \int_{0}^{x} \exp \left(-t^{2}\right) d t$. All these constraints are implemented in the following parametrization:

$$
\begin{aligned}
\lambda_{i \perp} & =\exp \tilde{\lambda}_{i \perp} \\
\lambda_{i \|} & =\exp \Delta \tilde{\lambda}_{i \|}+\frac{1}{2}\left(\lambda_{1 \perp}+\lambda_{2 \perp}\right) \\
f_{\{1, i s o\}} & =\frac{1}{2}\left(\operatorname{erf}\left(\tilde{f}_{\{1, i s o\}}\right)+1\right) \\
f_{2} & =1-f_{1}-f_{\text {iso }} \\
D_{\text {iso }} & =\exp \left(\tilde{D}_{\text {iso }}\right) .
\end{aligned}
$$

Employing this parameterization, the DW-signal [(1)] is described by the 11-dimensional parameter vector $\boldsymbol{\theta}$

$$
\boldsymbol{\theta}=\left\{\Delta \tilde{\lambda}_{1 \|}, \tilde{\lambda}_{1 \perp}, \Delta \tilde{\lambda}_{2 \|}, \tilde{\lambda}_{2 \perp}, \alpha_{1}, \alpha_{2}, \alpha_{3}, \alpha_{4}, \tilde{f}_{1}, \tilde{f}_{\text {iso }}, \tilde{D}_{\text {iso }}\right\} .
$$

The $\mathrm{FA}_{i}[38]$ of an axially symmetric tensor is given by

$$
\mathrm{FA}_{i}=\sqrt{\frac{\left(\lambda_{i \|}-\lambda_{i \perp}\right)^{2}}{\lambda_{i \|}^{2}+2 \lambda_{i \perp}^{2}}}=\frac{\lambda_{i \|}-\lambda_{i \perp}}{\sqrt{\lambda_{i \|}^{2}+2 \lambda_{i \perp}^{2}}} .
$$

\section{B. Maximum Likelihood Estimation}

The DWIs contain the magnitude of the complex MR signal. It is assumed that the constituting components are independently affected by Gaussian noise, such that the result is Rician distributed [39]. Consequently, the probability density function of a measured signal $S_{m, j}$ in a certain gradient direction $\mathbf{g}_{\mathbf{j}}$ with a chosen diffusion weighting $b_{j}$ is given by

$$
p\left(S_{m, j} \mid S_{\boldsymbol{\theta}, j}, \sigma\right)=\frac{S_{m, j}}{\sigma^{2}} \exp \left(-\frac{S_{m, j}^{2}+S_{\boldsymbol{\theta}, j}^{2}}{2 \sigma^{2}}\right) I_{0}\left(\frac{S_{m, j} S_{\boldsymbol{\theta}, j}}{\sigma^{2}}\right)
$$

with $S_{\boldsymbol{\theta}}, \boldsymbol{j}$ the true underlying value given the parameter vector $\boldsymbol{\theta}$ (and $\left\{\mathbf{g}_{\mathbf{j}}, b_{j}\right\}$ ), $\sigma$ the standard deviation of the noise and $I_{0}$ the zeroth order modified Bessel function of the first kind. Since the DWIs are independent, the joint probability density function $p\left(\mathbf{S}_{\mathbf{m}} \mid \boldsymbol{\theta}, \boldsymbol{\sigma}\right)$ of the signal profile $\mathbf{S}_{\mathbf{m}}$ in a voxel is given by the product of the marginal distributions for the measured signal $S_{m}$ in each of the $N_{g}$ diffusion weighted directions $\mathbf{g}_{\mathbf{j}}$

$$
p\left(\mathbf{S}_{\mathbf{m}} \mid \boldsymbol{\theta}, \boldsymbol{\sigma}\right)=\prod_{j=1}^{N_{g}} p\left(S_{m, j} \mid \boldsymbol{\theta}, \sigma\right) .
$$

However, given measurements $\mathbf{S}_{\mathbf{m}}$ and unknown $[\boldsymbol{\theta}, \sigma]$, the function $p\left(\mathbf{S}_{\mathbf{m}} \mid \boldsymbol{\theta}, \boldsymbol{\sigma}\right)$ is considered a likelihood function, indicated by $L\left(\boldsymbol{\theta}, \sigma \mid \mathbf{S}_{\mathbf{m}}\right)$. The Maximum Likelihood (ML) estimate of the parameters $\boldsymbol{\theta}$ is obtained by maximizing the $\log$ likelihood function: $\hat{\boldsymbol{\theta}}_{M L}=\arg \left\{\max _{\boldsymbol{\theta}}(\ln L)\right\}$. When the DWIs are assumed to be Rician distributed, the likelihood function is given by

$$
\begin{aligned}
& f(\boldsymbol{\theta}, \sigma)=\ln L\left(\boldsymbol{\theta}, \sigma \mid \mathbf{S}_{\mathbf{m}}\right)=-2 \ln (\sigma) \\
& \quad-\sum_{j=1}^{N_{g}}\left(\ln \left(S_{m, j}\right)-\frac{S_{m, j}^{2}+S_{\theta, j}^{2}}{2 \sigma^{2}}+\ln I_{0}\left(\frac{S_{m, j} S_{\boldsymbol{\theta}, \boldsymbol{j}}}{\sigma^{2}}\right)\right) .
\end{aligned}
$$

For high signal-to-noise ratio ( $\mathrm{SNR}=\mathrm{S}_{0} / \sigma>3$ [19]) the Rician distribution can be approximated by a Gaussian distribution. In that case the ML estimator reduces to a least-squares estimator.

The ML estimator has a number of favorable statistical properties. First, it can be shown that, under very general conditions, the ML estimator asymptotically achieves the CRLB, introduced in Section III-C) i.e., for an increasing value of $N_{g}$. Second, it can be shown that the ML estimator is consistent, which means that it asymptotically converges to the true value of the parameter in a statistically well-defined way [40]. Whether these properties also apply to a small number of observations can be assessed by experiments on synthetic (noise corrupted) data. 


\section{Cramér-Rao Analysis for Model Selection and Optimizing b}

Any chosen model and its parameterization $\boldsymbol{\theta}$ should be such that the parameters can be estimated precisely. Simultaneously, the acquired data should support the model to be fitted: the model, as described in Section II-A, is to be selected with respect to the precision in the estimation of $\boldsymbol{\theta}$. The dimensionality of $\boldsymbol{\theta}$ should be constrained in case the precision in specific parameters is low. In addition, the diffusion weighting $b$ influences the model fit (as argued in Section II-A) and needs to be carefully selected. For these purposes, we will adopt a Cramér-Rao analysis. The CRLB is the theoretical lower bound on the variance of any unbiased estimator of the parameters.

The Fisher information matrix is a measure of the amount of information about $\boldsymbol{\theta}$ that is present in the data. It is derived from the probability density function of the data [41], [42]

$$
\mathbf{I}=-\mathbb{E}\left[\frac{\partial^{2} \ln p\left(\mathbf{S}_{\mathbf{m}} \mid \boldsymbol{\theta}\right)}{\partial \boldsymbol{\theta} \partial \boldsymbol{\theta}^{\boldsymbol{T}}}\right]
$$

Diagonal elements of the inverse of the Fisher Information matrix $\mathbf{I}^{-1}$ reflect the variance of parameters, whereas off-diagonal elements relate to covariance between parameters. Effectively, the CRLB is a function for the curvature of the cost function, given by $\mathbf{I}^{-1}$. The CRLB-inequality yields [19]

$$
\operatorname{Cov}(\tau, \tau) \geq \operatorname{CRLB}=\left(\frac{\partial \boldsymbol{\tau}}{\partial \boldsymbol{\theta}}\right) \mathbf{I}^{-1}\left(\frac{\partial \tau}{\partial \boldsymbol{\theta}}\right)^{T} .
$$

Here, $(\partial \tau / \partial \boldsymbol{\theta})$ is the Jacobian of a potential transformation $\tau(\boldsymbol{\theta})$ of the parameter vector to a measure of interest, as explained in the appendix .

The CRLB implies that no unbiased estimator can be found for which the variance of the estimated parameters is lower than the diagonal elements of the CRLB matrix [40]. This inequality does not directly bound the off-diagonal elements, which specify the covariance between two parameters. It may be shown however, that the covariance of two ML-estimated parameters $\operatorname{cov}\left(\hat{\tau_{\mathrm{mML}}}, \hat{\tau_{\mathrm{nML}}}\right)$ will converge to the corresponding off-diagonal element CRLB $_{m n}$ when the number of samples goes to infinity [40].

The CRLB is quadratic in the parameters and is thus not directly comparable to the parameter values. We examine, therefore, the dimensionless relative CRLB (rCRLB), defined by

$$
\mathrm{rCRLB}_{\mathrm{mn}}=\sqrt{\frac{\left|\mathrm{CRLB}_{\mathrm{mn}}\right|}{\bar{\tau}_{m} \bar{\tau}_{n}}}
$$

where the indices $m, n$ denote the respective matrix element of CRLB and $\bar{\tau}_{m, n}$ the true parameter value.

The type of noise that affects the measurements needs to be properly addressed in the Fisher Information Matrix. For the Rician noise model the Fisher information matrix is given by [43]

$$
\mathbf{I}_{\mathbf{R}}=\frac{1}{\sigma^{2}} \sum_{j=1}^{N_{g}}\left(-\frac{S_{\boldsymbol{\theta}, \boldsymbol{j}}^{2}}{\sigma^{2}}+\mathbb{E}\left[\left(\frac{\mathbf{S}_{\boldsymbol{\theta}, \mathbf{j}} I_{1}\left(z_{j}\right)}{\sigma I_{0}\left(z_{j}\right)}\right)^{2}\right]\right) \cdot \frac{\partial S_{\boldsymbol{\theta}, \boldsymbol{j}}}{\partial \boldsymbol{\theta}} \frac{\partial S_{\boldsymbol{\theta}, \boldsymbol{j}}}{\partial \boldsymbol{\theta}^{\mathbf{T}}}
$$

with $z_{j}=S_{m, j} S_{\boldsymbol{\theta}, \mathbf{j}} / \sigma^{2}$ and $I_{1}$ a modified Bessel function of the first kind. The expected value can be calculated numerically
[43]. For high SNR, the noise distribution can be approximated by a Gaussian. Equation (11) then simplifies to

$$
\mathbf{I}_{\mathbf{G}}=\frac{1}{\sigma^{2}} \sum_{j=1}^{N_{g}}\left(\frac{\partial S_{\boldsymbol{\theta}, \boldsymbol{j}}}{\partial \boldsymbol{\theta}} \frac{\partial S_{\boldsymbol{\theta}, \boldsymbol{j}}}{\partial \boldsymbol{\theta}^{\boldsymbol{T}}}\right)
$$

\section{EXPERIMENTS}

First, we build the dual tensor model by analyzing which model parameters can be sufficiently precisely estimated and which need to be constrained (Section IV-A). Second, the optimal $\mathbf{b}$-values are determined for the chosen diffusion model in which we consider both a "homogeneous" and a "heterogeneous" sample, the latter containing a distribution of parameter values (Section IV-B). Cramér-Rao and Monte Carlo analyses allow us to do so. Third, in brain data FA-profiles are derived along fibers tracked through a crossing of white matter tracts in data of varying quality using different model parameterizations (Section IV-C).

\section{A. Model Selection}

We aim to build a dual tensor model such that its parameters can be estimated both precisely and accurately given a specific SNR and the number of diffusion encoding directions (see below). Different models of increasing complexity are proposed in Table I. For these models, we study how the axial and perpendicular diffusion parameters of the tensors need to be constrained to be estimated with low error. Also, the parameterization of the isotropic compartment is studied. Including such a compartment, for a single tensor model, yields a degenerated problem [44].

1) Maximal Precision: Cramér-Rao Analysis: The rCRLB, denoting the maximal precision by which parameters of interest $(\boldsymbol{\theta})$ can be estimated, is defined in (10). The upper limit for the rCRLB to be maintained throughout this paper is $15 \%$. This value is $\sim \sqrt{2}$ times larger than the $10 \%$ limit by which single tensor model parameters are estimated [20], since the length of our parameter vector is approximately twice that of [20].

The CRLB depends on the hypothetical true values of the parameters and as such representative parameters need to be defined. To determine representative diffusion values, we selected the anisotropic voxels having $\mathrm{FA}>0.5$ and $\mathrm{MD}>$ $1 \cdot 10^{-3} \mathrm{~mm}^{2} \mathrm{~s}^{-1}$ after a single tensor fit in a randomly selected dataset. The mean (diffusion) value and corresponding standard deviation were determined for the largest and smallest eigenvalues $\lambda_{1}$ and $\lambda_{3}$ ( $\lambda_{2}$ may be biased in crossing fiber voxels), and assigned to $\lambda_{1,2 \|}$ and $\lambda_{\perp}$. Notice that marginally perturbed values were chosen for $\lambda_{\perp, 1}$ and $\lambda_{\perp, 2}$. The volume fraction of the first tensor is $f_{1}=0.4$. The isotropic compartment is taken small $\left(f_{\text {iso }}=0.15\right)$, while the isotropic diffusion approximates the diffusivity of free water at $37^{\circ} \mathrm{C}: D_{\text {iso }}=3 \cdot 10^{-3} \mathrm{~mm}^{2} \mathrm{~s}^{-1}$. The representative parameters $\overline{\boldsymbol{\theta}}$ are given in Table I.

A $\mathbf{b}$-vector of length two $\mathbf{b}=\left[\begin{array}{ll}1.0 & 3.0\end{array}\right] \cdot 10^{3} \mathrm{~mm}^{-2} \mathrm{~s}$ is chosen, the selection of these values is addressed in the next section. The number of gradient directions $N_{q}$ is assumed constant: for each unique element of the b-vector an identical series of 92 three-fold tessellated icosahedric gradient directions is used. We adopt a modest $\mathrm{SNR}=25$. 

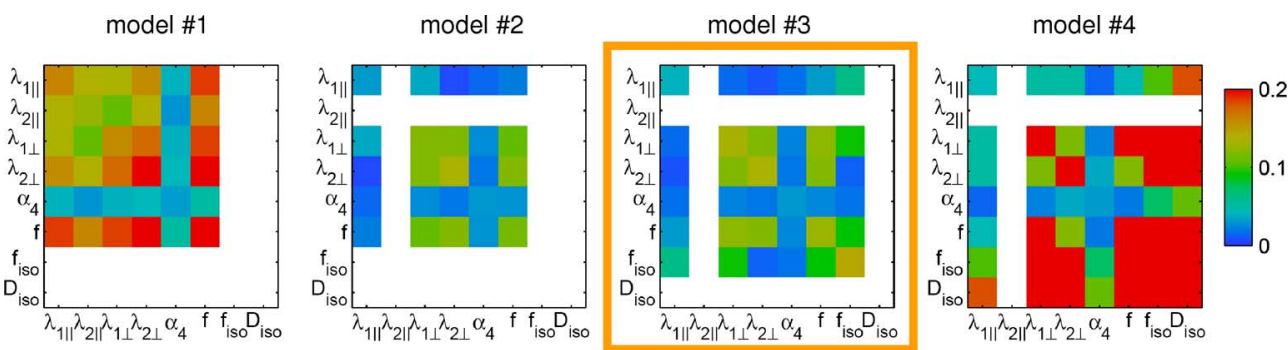

a
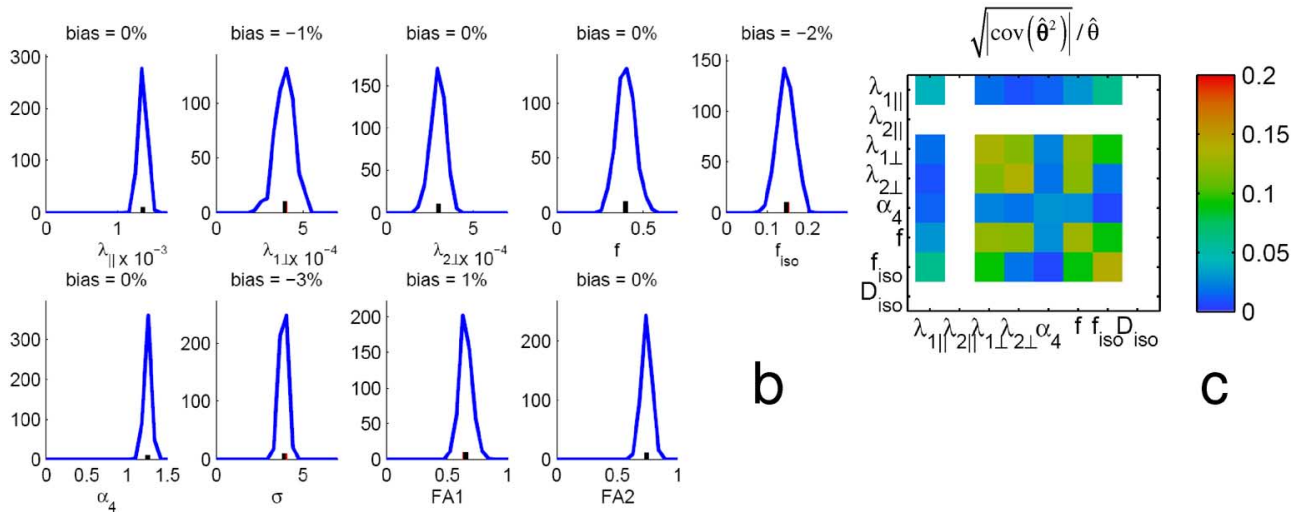

b

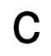

Fig. 1. (a) rCRLB-matrices for models involving different constraints (cf. Table I). The diagonals of these matrices contain the relative Cramer-Rao lowest bounds. Model \#3 is consistently used in our further analyses. Values above 0.2 are trimmed. (b) Distributions of estimated parameters determined in a Monte-Carlo experiment involving model \#3. The small black bars (at the bottom of the graphs) correspond to the true parameter values; relative biases are annotated. (c) Square root of the absolute covariance matrix emanating from the Monte Carlo experiment. The diffusion weighting was $\mathbf{b}=\left[\begin{array}{lll}1.0 & 3.0\end{array}\right] \cdot 10^{3} \mathrm{~mm}^{-2} \mathrm{~s}(\mathrm{Please}$ refer to the online version for illustration in color).

The calculated rCRLB-matrices for the four models (see Table I) are depicted in Fig. 1(a). The Cramér-Rao analysis reveals that even if $f_{\text {iso }}$ and $D_{\text {iso }}$ are constant $\lambda_{1,2 \|}$ cannot be estimated independently for the two tensors (model \#1): the values on the diagonal indicate rCRLB $>15 \%$ for all parameters except $\alpha_{4}$. We hypothesize that the imprecision in $\lambda_{1,2||}$ emanates from the high diffusion and hence the low signal measured along the axial orientation. In model \#2 $\lambda_{1 \|}$ and $\lambda_{2 \|}$ are set to be equal, while $\lambda_{1 \perp}$ and $\lambda_{2 \perp}$ are still included in the model. The second image in Fig. 1(a) shows that the constraint enables precise estimation of the remaining parameters. What is more, we find that constraining $\lambda_{1,2 \|}$ even allows for estimating $f_{\text {iso }}$ with sufficient precision ( $\mathrm{rCRLB}<15 \%$ ) as shown by the third image (model \#3): adding $f_{\text {iso }}$ does not significantly increase the CRLB of other parameters. The fourth image (model \#4) confirms that $f_{\text {iso }}$ and $D_{\text {iso }}$ cannot be estimated simultaneously.

2) Estimated Accuracy and Precision: Monte Carlo Simulation: Additionally, a Monte Carlo simulation is performed to study whether the theoretical lower bounds on the precision can be practically reached by the ML-estimator. Moreover, a potential bias and the uniqueness of the obtained ML-estimation are assessed. Parameter values are directly substituted into the diffusion model equation to generate signal values [(1)]. Subsequently, 500 of such simulated measurements are distorted by Rician noise. The ensembles of estimated parameter values for these measurements are expected to be Gaussian distributed [45].

The two-tensor model was implemented in Matlab (The MathWorks, Natick, MA). Levenberg-Marquardt optimiza- tion was used in all parameter estimations. The two-tensor model was initialized based on a single tensor estimation with three (sorted) eigenvalues $\lambda_{1 \ldots 3, s}$ and a rotation matrix $\mathbf{R}_{\text {single }}$ (without $\alpha_{4}$-term). We initialized anisotropic tensors by $\lambda_{1,2 \| \text { init }}=\lambda_{1 s}+\lambda_{2 s}, \lambda_{1,2 \perp \text { init }}=\lambda_{3 s}$, and with opening angle $\alpha_{4, \text { init }}=\arctan \left(\lambda_{2 s} / \lambda_{1 s}\right)$. The initial volume fractions were $f_{1 \text {,init }}=0.4$ and $f_{\text {iso, init }}=0.2$.

A Monte-Carlo experiment involving model \#3 rendered the histograms of the estimated parameter values depicted in Fig. 1(b). It shows that the distributions of the estimated parameters in each experiment are unimodal, while the bias is negligable (the maximal bias is $-3 \%$ in $\sigma$ ). This proves both a good initialization and the uniqueness of the solution.

The estimated relative standard deviations in the Monte-Carlo experiment approximate the rCRLBs: see Fig. 1(c). All estimated precisions were within $10 \%$ above the corresponding rCRLBs.

\section{B. Optimizing $\mathbf{b}$ for Different Tissue Configurations}

We aim to find the optimal $\mathbf{b}$ for estimating the diffusion shape of two crossing fiber tracts using the previously selected model. $S_{0}$ images (no diffusion weighting involved) are assumed to be acquired in all cases. Since the diffusion parameters are to be estimated with sufficient precision for two compartments, a vector with at least two supplementary nonzero values $\mathbf{b}$ is needed [5], in order to prevent the Fisher Information matrix from being rank deficient. Further, a larger vector $\mathbf{b}$ with a high range of values may be needed to adequately capture the heterogeneous diffusion in the data, as explained in Section II-B. The experiments for optimal $\mathbf{b}$ selec- 

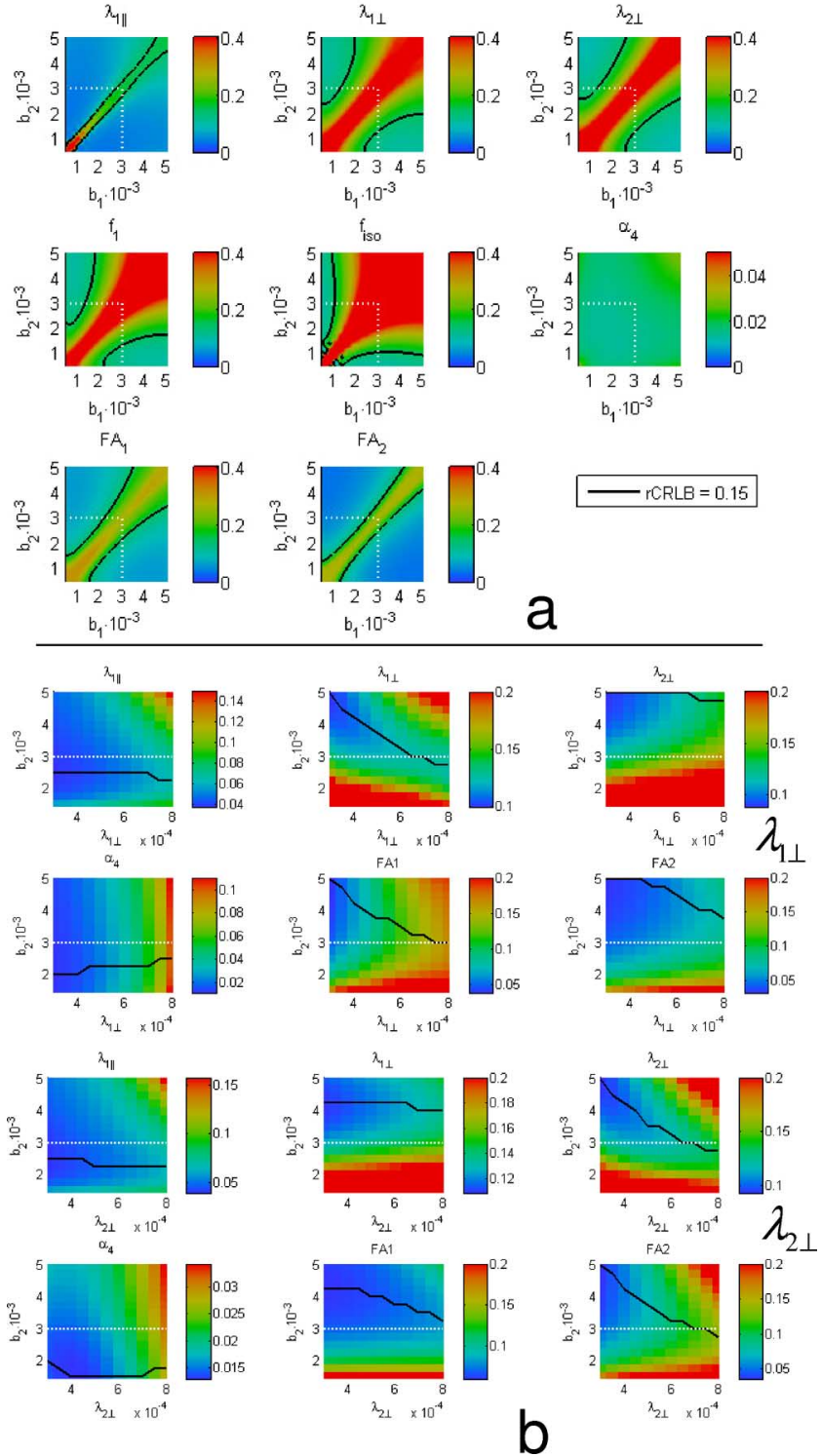

Fig. 2. (a) rCRLB-values for different parameters as a function of $b_{1,2}, \mathrm{SNR}=$ 25 . Values above 0.4 are trimmed. (b) The configurations in which the rCRLB is determined are based on $\overline{\boldsymbol{\theta}}$. (Table I) while adusting $\lambda_{1} \perp$ and $\lambda_{2} \perp$ respectively. Along the vertical axis the $b_{2}$-value is given, maintaining another nonzero $b$-value fixed at $b_{1}=1.0 \cdot 10^{3} \mathrm{~mm}^{-2} \mathrm{~s}$. (top) Horizontally $\lambda_{1 \perp}$ is varied while $\lambda_{2} \perp$ is constant. (bottom) $\lambda_{2} \perp$ is varied while $\lambda_{1} \perp$ is constant. Values above 0.2 are trimmed. The solid line in the figures give the $b_{2}$-value at which the rCRLB is minimal. The white dashed line denotes the upper-boundary of the region where the Gaussian diffusion assumption holds (see text).

tion focus on model \#3 (independent perpendicular diffusion $\lambda_{1,2 \perp}$ and $f_{\text {iso }}$ included).

1) Single Configuration: We will first determine the optimal $\mathbf{b}$ for a single configuration of crossing fibers $\overline{\boldsymbol{\theta}}$, see Table I. The selected model (see Section IV-A) is adopted, and rCRLBvalues will be computed for a two-element $\mathbf{b}$-vector. To minimize the rCRLB we also explore the high $b$-value range, although in Section II-B we argued that Gaussian diffusion cannot be assumed beyond $b>3.0 \cdot 10^{3} \mathrm{~mm}^{-2} \mathrm{~s}$. The optimum will be determined for $\mathrm{SNR}=25$.

Fig. 2(a) presents the rCRLB for the mean parameter vector $\overline{\boldsymbol{\theta}}$ (Table I) for varying supplementary $\mathbf{b}$-values (along the axes). Note that values on the diagonals effectively involve merely one $b$-value. A detailed inspection of the results presented in Fig. 2(a) shows that the $\mathrm{rCRLB}_{\mathrm{FA}_{1,2}}$ are minimal at $\mathbf{b}=\left[\begin{array}{lll}0.75 \ldots 1.0 & 3.0 \ldots 4.25\end{array}\right] \cdot 10^{3} \mathrm{~mm}^{-2} \mathrm{~s}$, with $\operatorname{rCRLB}_{\mathrm{FA}_{1}}=6 \ldots 7 \%$. The rCRLB of all parameters remain close to their optimal rCRLB around these b-values.

2) Varying Perpendicular Diffusion: Then, the optimal $\mathbf{b}=\left[\begin{array}{ll}b_{1} & b_{2}\end{array}\right]$ is determined for varying perpendicular diffusion values $\lambda_{1,2 \perp}$ involved in the model configuration $\overline{\boldsymbol{\theta}}$. rCRLB-values are computed for $\lambda_{1,2 \perp}=$ $\left[\begin{array}{lll}0.3 & \ldots & 0.8\end{array}\right] \cdot 10^{-3} \mathrm{~mm}^{2} \mathrm{~s}^{-1}$. Simultaneously, the $b_{2}$-value is varied, taking the $b_{1}$-value fixed at $b_{1}=1.0 \cdot 10^{3} \mathrm{~mm}^{-2} \mathrm{~s}$. If we observe significant variation in rCRLB at the location of the minimum, this can be considered to be an indication that multiple $b_{1,2}$-values are needed to model the entire diffusion range with low error.

The rCRLBs under these circumstances are depicted in Fig. 2(b). The rCRLB at optimal $b_{2}$ are not significantly different from the rCRLB at constant $b_{2}=\left[\begin{array}{lll}2.5 & \ldots & 3.0\end{array}\right] \cdot 10^{3} \mathrm{~mm}^{-2} \mathrm{~s}$, which is about the optimum for the entire parameter vector. Only $\mathrm{rCRLB}_{\lambda_{1,2 \perp}}$ slightly improves from $14 \%$ $\left(b_{2}=3.0 \cdot 10^{3} \mathrm{~mm}^{-2} \mathrm{~s}\right)$ to $10 \%\left(b_{2}=5.0 \cdot 10^{3} \mathrm{~mm}^{-2} \mathrm{~s}\right)$ for a low diffusion value $\lambda_{1,2 \perp}=0.3 \cdot 10^{-3} \mathrm{~mm}^{2} \mathrm{~s}^{-1}$.

3) "Heterogeneous" Sample: Third, we looked into the number of values of $\mathbf{b}$ needed to measure a "heterogeneous" sample precisely and accurately, by considering a series of different crossing fiber configurations. The mean parameter values $\overline{\boldsymbol{\theta}}$ and corresponding standard deviations in Table I are at the basis to generate 100 Gaussian distributed parameter values.

In all these experiments, the total number of DWIs stays constant. This is realized by randomly assigning an equal number of gradient directions to each supplementary $b$-value. Optimizing this distribution over $\mathbf{b}$ is beyond the scope of this paper. $b$-values in the range of $\left[\begin{array}{lll}1.0 & \ldots & 5.0\end{array}\right] \cdot 10^{3} \mathrm{~mm}^{-2} \mathrm{~s}$ are considered. Note that Gaussian diffusion can be safely assumed only if $b<3.0 \cdot 10^{3} \mathrm{~mm}^{-2} \mathrm{~s}$ [31].

The mean rCRLB $_{\mathrm{FA}}$ (averaged for both tensors) is determined using all 100 configurations for an increasing length of $\mathbf{b}$. The selected $\mathbf{b}$ and the corresponding mean rCRLB are given in Table II. The table shows that the mean rCRLB remained constant when employing a $\mathbf{b}$ with more than two values. This experiment shows that $\mathbf{a} \mathbf{b}$ of length two is also sufficient to reach the smallest rCRLB over a range of expected parameter values.

Additionally, a Monte-Carlo experiment is performed by simulating measurements of these configurations that are distorted by Rician noise to yield 100 noise realizations per parameter configuration. The bias, standard deviation and root mean squared error (RMSE) in FA and MD (averaged for both tensors) are computed for each combination of $\mathbf{b}$.

The outcome of the Monte-Carlo experiment is given in Fig. 3. This figure shows that the optimal value of $\mathbf{b}$ to be chosen for a heterogeneous sample is $\mathbf{b}=\left[\begin{array}{ll}1.0 & 3.5\end{array}\right] \cdot 10^{3} \mathrm{~mm}^{-2} \mathrm{~s}$, because for these values of $\mathbf{b}$ the RMSE in both FA and MD is minimal. The RMSE balances between a minimum in the bias at a lower $\mathbf{b}=\left[\begin{array}{ll}1.0 & 2.5\end{array}\right] \cdot 10^{3} \mathrm{~mm}^{-2} \mathrm{~s}$ and the standard deviation at a slightly higher $\mathbf{b}=\left[\begin{array}{ll}1.0 & 4.0\end{array}\right] \cdot 10^{3} \mathrm{~mm}^{-2} \mathrm{~s}$ respectively. $n_{\mathbf{b}}=2$ is the optimal number of elements in $\mathbf{b}$, since RMSE(FA) remains constant for $n_{\mathrm{b}}>2$. The relative 
TABLE II

b-VECTOR YIELDING A MiNIMUM MEAN RCRLB ON THE FA AND A MiNIMUM MEAN RMSE ON THE FA, ON CONFIGURATIONS DRAWN FROM TABLE I. DifFERENT $b$-VALUE CONFIGURATIONS, As DESCRIBED IN SECTION IV-B, Were USED. NOte That IN THE CRAmÉR-RAO ANALYSIS A SUb-OPTIMAL RESUlT WiTHIN THE GAUSSIAN DIFFUSION RANGE (*) IS INCLUDED

\begin{tabular}{l|llll}
\hline \multicolumn{5}{|c}{ Cramér-Rao analysis } \\
\hline \hline rCRLB ${ }_{\mathrm{FA}}$ & $\mathbf{b} \cdot 10^{3} \mathrm{~mm}^{-2} \mathrm{~s}$ & & \\
\hline \hline 0.26 & 5.0 & & & \\
\hline 0.065 & 0.75 & 4.5 & & \\
\hline $0.073^{*}$ & 0.75 & $3.0^{*}$ & & \\
\hline 0.064 & 0.75 & 4.25 & 4.5 & \\
\hline 0.065 & 0.75 & 0.75 & 4.0 & 4.75 \\
\hline
\end{tabular}

\begin{tabular}{l|llll}
\hline \multicolumn{5}{c}{ Monte-Carlo simulation } \\
\hline \hline RMSE(FA) & $\mathbf{b} \cdot 10^{3} \mathrm{~mm}^{-2} \mathrm{~s}$ & \\
\hline \hline 0.23 & 3.0 & & & \\
\hline 0.074 & 1.0 & 3.5 & & \\
\hline & & & & \\
\hline 0.074 & 0.5 & 1.0 & 3.5 & \\
\hline 0.074 & 0.5 & 0.5 & 1.0 & 3.5 \\
\hline
\end{tabular}

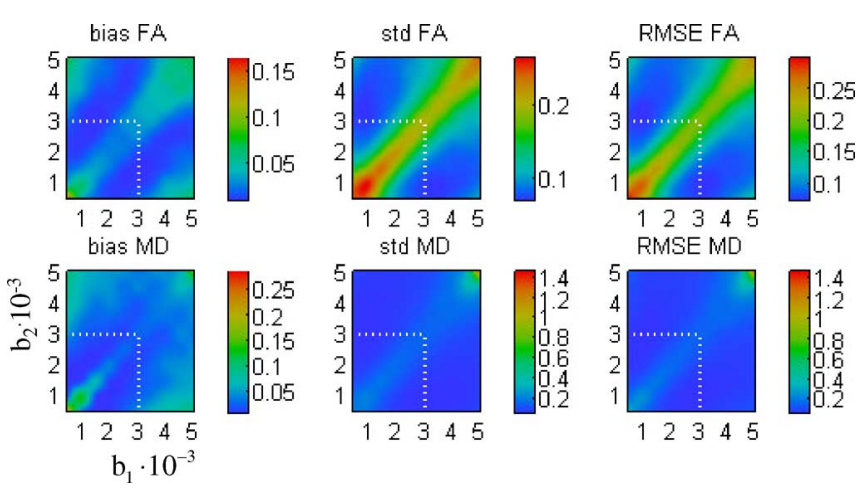

Fig. 3. Bias in the estimated parameter value (left), the corresponding standard deviations (middle) and the root mean squared error, all averaged over both tensors, on simulated measurements (drawn from the distributions indicated in Table I). The axes correspond to the $b$-values at which this Monte-Carlo experiment was performed. The white dashed line denotes the upper-boundary of the region where the Gaussian diffusion assumption holds.

standard deviation closely approaches the rCRLB determined in Table II. Also, notice the high standard deviation along the diagonal (reflecting the usage of merely one $b$-value), which is in accordance with the Cramér-Rao analysis.

\section{Validation in Brain Data}

A quantitative validation of estimated diffusion properties within fiber crossings in the human brain is complicated by the lack of a ground truth. To the best of our knowledge, a phantom with known diffusion properties (apart from fiber orientations [46]) is unavailable. We have chosen to track fibers through a crossing fiber region, where gyral projections of the corpus callosum (commissural fibers) are crossing the corticospinal tract. FA-profiles along the tracts will be compared for data acquired with different $\mathbf{b}$.

1) Data Acquisition and Preprocessing: Diffusion Weighted Image acquisition of 20 axial slices (mid-brain) of a healthy volunteer was performed on a Philips Intera 3.0T MRI scanner (Philips Intera, Philips Healthcare, Best, The Netherlands) by means of a spin echo EPI-sequence. An imaging matrix of $112 \times 110$ was used, from which a $128 \times 128$ sized slices were reconstructed, with a voxel size of $1.7 \times 1.7 \times 2.2 \mathrm{~mm}^{3}$. An echo time of $\mathrm{TE}=84 \mathrm{~ms}$ and repetition time of $\mathrm{TR}=3800 \mathrm{~ms}$ were used. The diffusion weighting was along 92 three-fold tessellated icosahedric gradient directions, with five $b$-values: $\mathbf{b}=\left[\begin{array}{lllll}1.0 & 1.4 & 1.8 & 2.2 & 3.0\end{array}\right] \cdot 10^{3} \mathrm{~mm}^{-2} \mathrm{~s}$. Per $b$-value, one non-diffusion weighted image $S_{0}$ was acquired. 20 axial slices were acquired, resulting in a total scanning time of $30 \mathrm{~min}$. Deformation induced by eddy currents was corrected for using an affine registration in the phase encoding direction [47]. In addition, a rigid registration of the $S_{0}$-images and coregistration of the DWIs corrected for patient head motion (up to 2 voxels). One average $S_{0}$-image was computed and used in all experiments with subsets of $b$-values. Subsequently, Rician noise in the data was reduced using an adaptive linear minimum mean square estimator (LMMSE) [24], [48]. This filtering approach uses an estimate of the noise level $\sigma . \sigma$ is estimated per voxel by fitting a dual tensor model which does not include an isotropic compartment: model \#2 from Table I.

2) Consistent Parameter Estimation: From the acquired DWIs, different data sets with a two-element $\mathbf{b}$-vector were generated. From the antipodally symmetric set of gradient-directions, one gradient direction was randomly chosen and assigned to the first $b_{1}$-value. Simultaneously, the antipodal gradient direction was assigned to the second $b_{2}$-value, resulting in 46 gradients per $b$-value. In this way, the angular resolution over all permutations of $\mathbf{b}$ was equal. In addition, the entire scanned dataset-involving a five-element $\mathbf{b}$-vector and all DWIs - was used to assess an improved FA-estimation when sampling a broader range of diffusion values. The dual-tensor model was fit to all created datasets. The estimated perpendicular diffusivity and $\mathrm{FA}_{1,2}$-values are sorted into $\lambda_{\min , \perp}$ and $\lambda_{\max , \perp}$ and $\mathrm{FA}_{\{\min , \max \}}$, respectively.

Model \#3 was estimated taking constant $b_{1}=1.0$. $10^{3} \mathrm{~mm}^{-2} \mathrm{~s}$ and varying $b_{2}$-values. Fig. 4(a) shows axial slices depicting the parameter values estimated on data involving $\mathbf{b}=\left[\begin{array}{ll}1.0 & 3.0\end{array}\right] \cdot 10^{3} \mathrm{~mm}^{-2}$ s. Notice that there is high inter-voxel "consistency." The $\lambda_{\|}$image displays good contrast between white and gray matter, whereas the $f_{\text {iso-image mainly }}$ highlights the CSF. Isolated black voxels may be observed in the $\lambda_{\min , \perp}$ image. These correspond to samples containing only a single anisotropic compartment. Here $\lambda_{\min , \perp}$ and $\mathrm{FA}_{\{\text {min,max }\}}$ are undefined since the volume fraction $f$ of the corresponding tensor approximates zero.

We adopted a grid approach to perform the experiments using the infrastructure of the Dutch Virtual Laboratory for Medical Image Analysis [49]. Thus, computing times were reduced from one week (estimated) to $6 \mathrm{~h}$, a 28:1 improvement in speed.

3) FA-Profiles Along Crossing Fiber Tracts: Fiber tracking was performed using High Angular Fiber Tracking [50]. As an extension to common streamline tracking algorithms, this method generates branches of fibers if, within a voxel, the angle between both tensors exceeds $12.5^{\circ}$. The curvature threshold was set to $20^{\circ}$ per voxel, and the single tensor FA threshold yielded $\mathrm{FA}_{\text {single }}>0.1$. One seeding region of interest (ROI) and one additional ROI through which fibers should pass were defined. Fibers were tracked through the crossing of the corticospinal tract with gyral projections of the corpus callosum (commissural fibers). The corticospinal tract was only partially 

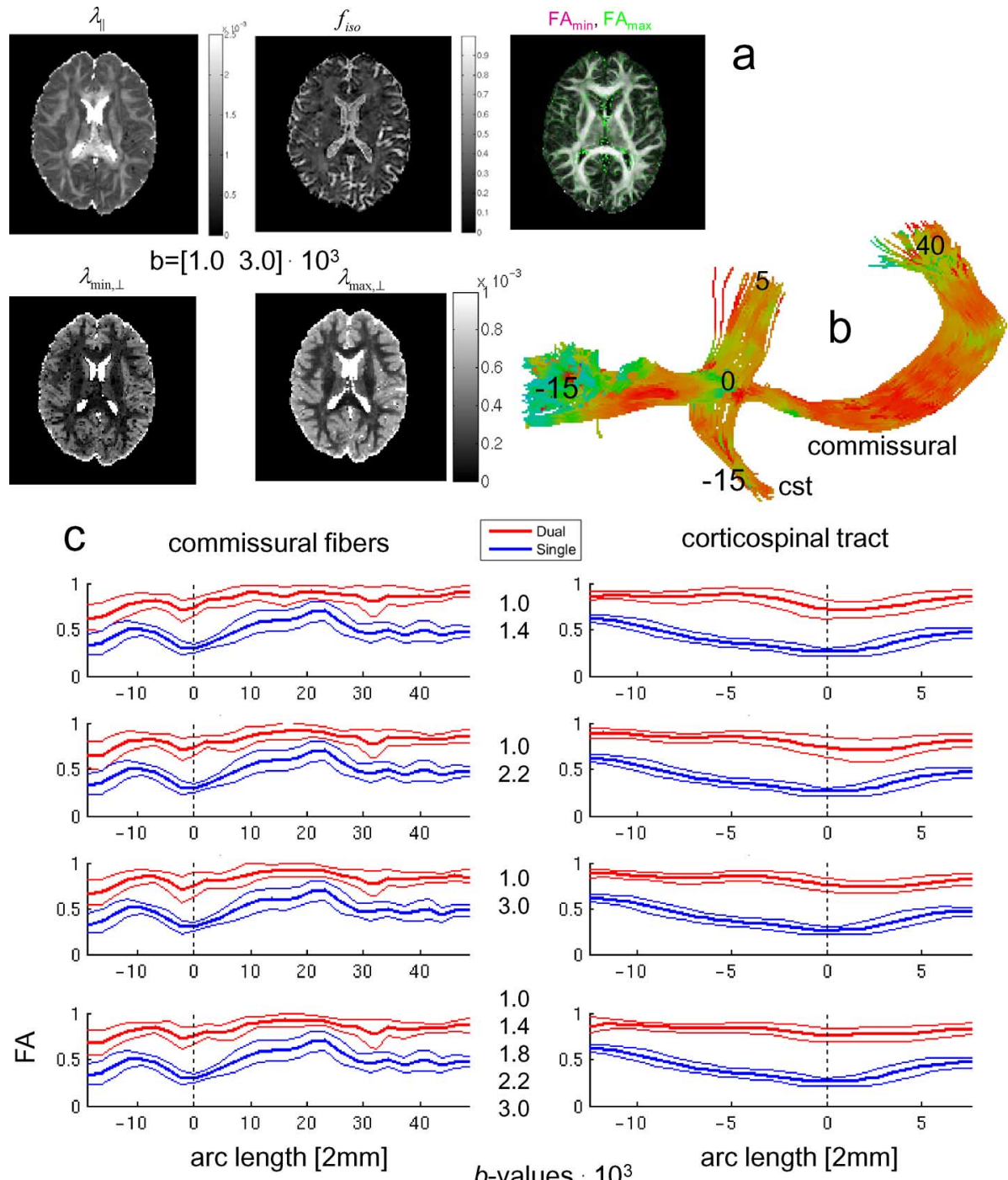

Fig. 4. Results in brain data. (a) $\lambda_{\|}, \lambda_{\min , \max , \perp}, f_{\text {iso }}$ and $\mathrm{FA}_{\{\min , \max \}}$ estimated in one axial slice, $\mathbf{b}=[1.0 \quad 3.0] \cdot 10^{3}$ mm ${ }^{-2} \mathrm{~s}$. $\mathrm{FA}\{$ min, $\max \}$ are colored magenta and green respectively, such that white colored voxels reflect $\mathrm{FA}_{\min }=\mathrm{FA}_{\max }$ and green voxels $\mathrm{FA}_{\min }<\mathrm{FA}$ max (b) Tracked fibers: the corticospinal tract (CST) and commissural fibers. Color denotes the FA. (c) Mean FA-profiles (in bold) with standard deviations calculated through dual (red) and single (blue) tensor fits as a function of the arc length (in units of $2 \mathrm{~mm}$ ) of the commissural fibers (left) and the corticospinal tract (right). The data was acquired at different $b$-value configurations as annotated. The crossing is indicated with a dashed line and is considered the origin.

tracked, due to the limited scanning region; it was confirmed that the crossing region was completely present in the data, though. Two ROIs per tract sufficed to successfully track the fibers.

During tracking, the most closely aligned tensor per voxel is selected. Along the tracked fibers, FA-profiles are computed using the aligned tensors. The mean and standard deviation of the FA in voxels through which fibers traverse are estimated in the plane perpendicular to the centerline of the tracts. At the location of the fiber crossing, oblate single tensors are expected whose FA is biased, i.e., lower than the FA-values of the prolate dual tensors. Dual-tensor FA-profiles are higher (i.e., less biased) than their single-tensor counterparts at the location of the fiber crossing. An additional global offset in the FA may be apparent due to the included isotropic fraction in the dual tensor model. In addition, there should be spatial consistency in FA in adjacent single- and dual-tensor regions.

Fig. 4(b) shows the corticospinal and commissural fibers tracked in this data. The FA-profiles along those tracts are displayed in Fig. 4(c) in which we estimate parameters for two models: a single tensor model and dual tensor model \#3. At the position of the crossing, the bias of the single tensor model is most distinct. It can also be seen that dual tensor FA-profiles are consistent at the transition of a single to a dual orientation region, whereas single tensor profiles show a reduction in FA. Additionally, the standard deviation of the estimated FA slightly decreases for higher $b_{2}$-values, particularly in the corticospinal tract, and is minimal for $\mathbf{b}=\left[\begin{array}{ll}1.0 & 3.0\end{array}\right] \cdot 10^{3} \mathrm{~mm}^{-2} \mathrm{~s}$. Notice that the dual tensor FA-profiles are systematically higher than the single tensor profiles (also remote from the crossing). This effect emanates from the inclusion of an isotropic component in the model. Finally, estimating the model with a five-element b-vector renders only marginally different profiles.

\section{DISCUSSION}

A framework was presented for model selection and optimization of acquisition parameters for estimating diffusion 
properties in fiber crossings. A dual tensor model was studied for which the optimal diffusion weighting $\mathbf{b}$ was determined. The framework is directly applicable to other parameterized diffusion models and different experimental conditions.

\section{A. Model Selection}

The Cramér-Rao analysis showed that at a modest SNR the perpendicular diffusion $\lambda_{1,2 \perp}$ can be precisely estimated in model \#3, whereas the axial diffusions $\lambda_{1,2 \|}$ cannot be independently modeled, since they result in a large rCRLB in model \#1. This may be explained by higher DW-signal attenuation along the axial orientation, which has a higher diffusion and thus a lower $\mathrm{SNR}_{\mathrm{DW}}$. In other words, our inability to independently estimate $\lambda_{1,2 \|}$ is a limitation of the DW-MRI modality under the described circumstances. The strength of our work is in independently capturing myelin degradation in crossings. Histology in ischemic mice confirms myelin degradation and because of that increased mobility of the water molecules perpendicular to the axons, measured by DW-MRI as an increased $\lambda_{\perp}[3]$.

An isotropic compartment was modeled by a single parameter in model \#3. In fact, it was shown that adding the isotropic compartment to model \#2 does not reduce the precision of the other parameters. Simultaneously estimating $f_{\text {iso }}$ and $D_{\text {iso }}$ led to a degeneration in model \#4 [Fig. 1(a)].

In previous work, particularly the angular resolution attracted attention. An angle of $\pi / 4$ between the tensors could be accurately determined by a model that only estimated orientations, (the experiment involved a single $b=2000 \mathrm{~mm}^{-2} \mathrm{~s}$ ) [33]. $Q$-ball imaging and the Diffusion Orientation Transform also achieved an angular resolution of $\pi / 4$ [51]. The experiments that we have performed involve a lower angular resolution $\left(2 \cdot \alpha_{4}=\pi / 2.5\right)$ since our main interest is to ensure a low error in the estimated diffusivity parameters. Lower values of $\alpha_{4}$ will e.g., result in $\mathrm{rCRLB}_{\mathrm{FA}}>0.15$ under equal measurement conditions.

The Monte Carlo simulation showed that the estimator converged to a unique solution. Moreover, the observed precisions were close to the CRLB, which demonstrates a good estimator performance. Simultaneously estimating the diffusion "size" and "shape" parameters was previously reported to be a difficult problem [8]. The eigenvalue parameterization [(2)] prevents degeneration of the parameter estimation.

The dual tensor model (\#3) was estimated independently per voxel. In other work, the spatial continuity of the parameters was included as a constraint [52]. The maximal precision to be achieved in this approach might also be quantified by means of our Cramér-Rao framework. The framework is directly applicable to multitensor approaches [9]-[11], higher order tensors [12], constrained spherical deconvolution [13] or a series expansion of the PDF made of Gaussian-Laguerre and Spherical Harmonics functions [53], which all are parametric models. Additionally, the ML-approach could be extended to a Bayesian estimation scheme by incorporating prior distributions on parameters [33].

\section{B. Optimizing b for Different Tissue Configurations}

The Cramér-Rao analysis also showed that a two-element b-vector is needed for independently estimating axial and perpendicular diffusion as well as an isotropic compartment [model \#3, Fig. 2(a)]. This result confirms previous statements [5]. A minimal $\mathrm{rCRLB} \mathrm{FA}_{1}=6.5 \%$ was found for $\mathbf{b}=\left[\begin{array}{ll}0.75 & 4.5\end{array}\right]$. $10^{3} \mathrm{~mm}^{-2} \mathrm{~s}$, which only marginally raised $\mathrm{rCRLB}_{\mathrm{FA}_{1}}=7.5 \%$ for $\mathbf{b}=\left[\begin{array}{ll}0.75 & 3.0\end{array}\right] \cdot 10^{3} \mathrm{~mm}^{-2} \mathrm{~s}$ within the Gaussian diffusion range. In the Monte Carlo analysis, the RMSE was minimal at a lower $\mathbf{b}=\left[\begin{array}{ll}1.0 & 3.5\end{array}\right] \cdot 10^{3} \mathrm{~mm}^{-2} \mathrm{~s}$, due to an increasing bias at higher $\mathbf{b}$-values. Notice that the precisions were well below the maximally allowed value, e.g., $\mathrm{CRLB}_{\mathrm{FA}}=6.5 \%<15 \%$.

We observed that the minimum in rCRLB and RMSE as a function of $\mathbf{b}$ is rather "shallow" (see e.g., Fig. 2). Accordingly, the results on brain data were fairly robust with respect to modification of $b_{2}$. A significantly higher bias in the FA for low $b$-values $\left(b=1.0 \cdot 10^{3} \mathrm{~mm}^{-2}\right.$ s, see Fig. 3$)$ is in agreement with earlier findings [35].

The $\mathbf{b}$ associated with a minimum rCRLB did not vary as $\lambda_{\perp}$ was changed [Fig. 2(b)]. Moreover, it was shown that two elements in $\mathbf{b}$ suffice for estimating the parameters in a heterogeneous sample: rCRLB $_{\mathrm{FA}}$ and RMSE(FA) did not decrease when $\mathbf{a}$ b up to length four was used. In previous work, the optimal $b$-value was shown to be mainly dependent on the mean diffusivity [8], which is in contrast to our findings. We attribute this to a difference in model selection: Alexander and coworkers constrained the diffusivity values to be equal for both tensors. A single nonzero $b$-value then suffices for estimating the diffusion. This value appears to be more sensitive to the expected diffusivity values. Our independent parameterization of the perpendicular diffusivity minimizes bias and variance in the parameters when the distance between the $b$-values is large. This allows us to independently estimate the FA in both compartments.

It may be expected that the optimal $b$-values found in the Cramér-Rao analysis correspond to the underlying diffusivity scales. The inverse diffusion weighting values $1 / b_{1}=1 \cdot 10^{-3}$ and $1 / b_{2}=0.3 \cdot 10^{-3}$ indicate that lower diffusion values are emphasized (we used diffusion values of around $1.5 \cdot 10^{-3} \mathrm{~mm}^{2} \mathrm{~s}^{-1}$ and $0.4 \cdot 10^{-3} \mathrm{~mm}^{2} \mathrm{~s}^{-1}$ in the model of the experiments). Optimization of acquisition parameters previously resulted in $b \sim 2.2-2.8 \cdot 10^{3} \mathrm{~mm}^{-2} \mathrm{~s}$ [8], which is close to our optimum for $b_{2}$.

\section{Validation in Brain Data}

Model \#3 was used to estimate the diffusion shape in the crossing of the corticospinal tract and the corpus callosum. It yielded a "consistent" FA-profile at the location of the crossing, whereas single tensor profiles showed a reduction in FA. The standard deviation of the estimated FA was minimal for $\mathbf{b}=$ $\left[\begin{array}{ll}1.0 & 3.0\end{array}\right] \cdot 10^{3} \mathrm{~mm}^{-2}$ s. It was demonstrated that the FA profiles for $n_{\mathbf{b}}=2$ did not differ significantly from the FA-profiles emanating from $\mathbf{b}$ with more $n_{\mathbf{b}}=5$ elements.

FA-values estimated in brain data were systematically lower for the single tensor model than for a two tensor model even in noncrossing tissue regions (Fig. 4). We attribute this to the isotropic compartment that is lacking in the single tensor model. Previous work considered the relative proportion of anisotropic signal rather than the FA per tensor [54]. Note that exploring 
data-driven model selection, such as automatic relevance detection [33] is beyond the scope of this work.

This work defines fundamental limits for comparative studies to correctly analyze complex white matter structures.

\section{APPENDIX}

Derivatives to Estimated Parameters: The performance of the Levenberg-Marquardt optimization is significantly increased by including derivatives of the signal to the parameters $\partial S_{\boldsymbol{\theta}}(\boldsymbol{\theta}) / \partial \boldsymbol{\theta}$. To obtain the derivatives to our reparameterization, we first write

$$
\begin{aligned}
\frac{\partial S_{j}}{\partial \lambda_{i \|}}= & -b_{j} \mathbf{g}_{\mathbf{j}}^{\mathbf{T}} \mathbf{R}_{\mathbf{i}} \operatorname{diag}\left(\begin{array}{lll}
1 & 0 & 0
\end{array}\right) \mathbf{R}_{\mathbf{i}}^{\mathbf{T}} \mathbf{g}_{\mathbf{j}} f_{i} S_{i j} \\
\frac{\partial S_{j}}{\partial \lambda_{i \perp}}= & -b_{j} \mathbf{g}_{\mathbf{j}}^{\mathbf{T}} \mathbf{R}_{\mathbf{i}} \operatorname{diag}\left(\begin{array}{lll}
0 & 1 & 1
\end{array}\right) \mathbf{R}_{\mathbf{i}}^{\mathbf{T}} \mathbf{g}_{\mathbf{j}} f_{i} S_{i j} \\
\frac{\partial S_{j}}{\partial \alpha_{j}} & =-b_{j} \sum_{i=1,2} \mathbf{g}_{\mathbf{j}}^{\mathbf{T}}\left(\frac{\partial \mathbf{R}_{\mathbf{i}}}{\partial \alpha_{\mathbf{j}}} \mathbf{E}_{\mathbf{i}} \mathbf{R}^{\mathbf{T}}+\mathbf{R}_{\mathbf{i}} \mathbf{E}_{\mathbf{i}} \frac{\partial \mathbf{R}_{\mathbf{i}}^{\mathbf{T}}}{\partial \alpha_{j}}\right) \mathbf{g}_{\mathbf{j}} f_{i} S_{i j} \\
& j \in\{1 \ldots 4\} \\
\frac{\partial S_{j}}{\partial f_{1}}= & S_{1 j}-S_{2 j} \\
\frac{\partial S_{j}}{\partial f_{\text {iso }}}= & S_{\text {iso }}-S_{2 j} \\
\frac{\partial S_{j}}{\partial D_{\text {iso }}} & =-b_{j} f_{\text {iso }} S_{\text {iso }} .
\end{aligned}
$$

Note that $f_{2}=1-f_{1}-f_{\text {iso. }}$. The rotation matrices for both tensors are written as

$$
\begin{gathered}
\mathbf{R}_{\mathbf{i}}=\left[\begin{array}{ccc}
1 & 0 & 0 \\
0 & \cos \alpha_{1} & -\sin \alpha_{1} \\
0 & \sin \alpha_{1} & \cos \alpha_{1}
\end{array}\right] \cdot\left[\begin{array}{ccc}
\cos \alpha_{2} & 0 & \sin \alpha_{2} \\
0 & 1 & 0 \\
-\sin \alpha_{2} & 0 & \cos \alpha_{2}
\end{array}\right] \\
\cdot\left[\begin{array}{ccc}
\cos \left(\alpha_{3}+(-1)^{i} \alpha_{4}\right) & -\sin \left(\alpha_{3}+(-1)^{i} \alpha_{4}\right) & 0 \\
\sin \left(\alpha_{3}+(-1)^{i} \alpha_{4}\right) & \cos \left(\alpha_{3}+(-1)^{i} \alpha_{4}\right) & 0 \\
0 & 0 & 1
\end{array}\right]
\end{gathered}
$$

from which derivatives $\partial \mathbf{R}_{\mathbf{i}} / \partial \alpha_{j}$ are obtained and substituted in (A.15). The computed signal per tensor $i$ equals

$$
S_{i j}=S_{0} \exp \left(-b_{j} \mathbf{g}_{\mathbf{j}}^{\mathbf{T}} \mathbf{D}_{\mathbf{i}} \mathbf{g}_{\mathbf{j}}\right) .
$$

Derivatives to our parameterization then are

$$
\begin{aligned}
\frac{\partial S_{j}}{\partial \Delta \tilde{\lambda}_{i \|}} & =\frac{\partial S_{j}}{\partial \lambda_{i \|}} s \exp \Delta \tilde{\lambda}_{i \|} \\
\frac{\partial S_{j}}{\partial \tilde{\lambda}_{i \perp}} & =\left\{\frac{\partial S_{j}}{\partial \lambda_{i \perp}}+\frac{1}{2}\left(\frac{\partial S_{j}}{\partial \lambda_{1 \|}}+\frac{\partial S_{j}}{\partial \lambda_{2 \|}}\right)\right\} s \exp \lambda_{i \perp} \\
\frac{\partial S_{j}}{\partial \tilde{f}_{\{1, i s o\}}} & =\frac{1}{\sqrt{\pi}} \exp \left(-\left(\tilde{f}_{\{1, i s o\}}\right)^{2}\right) \\
\frac{\partial S_{j}}{\partial \tilde{D}_{\text {iso }}} & =\frac{\partial S_{j}}{\partial D_{\text {iso }}} \exp \tilde{D}_{\text {iso }}
\end{aligned}
$$

with $s=10^{3}$ a constant scaling factor to achieve approximately equal order of magnitude for all parameter values in numerical optimization.
Cramér-Rao Lower Bounds on Eigenvalues and FA: We will now derive the CRLB on eigenvalues and FA, as mentioned in Section III-C. Exponential and error function mappings are ignored in calculating the CRLB, since they merely aim to constrain the optimization. Equation (2) then reduces to a parametrization of the axial diffusivity

$$
\lambda_{i \|}=\Delta \tilde{\lambda}_{i \|}+\frac{1}{2}\left(\lambda_{1 \perp}+\lambda_{2 \perp}\right)
$$

and an adapted parameter vector $\boldsymbol{\theta}^{\prime}$

$$
\boldsymbol{\theta}^{\prime}=\left\{\Delta \tilde{\lambda}_{1 \|}, \lambda_{1 \perp}, \Delta \tilde{\lambda}_{2 \|}, \lambda_{2 \perp}, \alpha_{1}, \alpha_{2}, \alpha_{3}, \alpha_{4}, f_{1}, f_{\text {iso }}, D_{\text {iso }}\right\} \text {. }
$$

Equation (9) describes the CRLB-inequality including $\left(\partial \tau / \partial \boldsymbol{\theta}^{\prime}\right)$, which is the Jacobian of a potential transformation $\tau\left(\boldsymbol{\theta}^{\prime}\right)$ of the parameter vector to a measure of interest. For the purpose of calculating the CRLBs of the eigenvalues, such as $\mathrm{CRLB}_{\lambda_{1 \|}}=\left(\partial \lambda_{1 \|} / \partial \boldsymbol{\theta}^{\prime}\right) \mathbf{I}^{-1}\left(\partial \lambda_{1 \|} / \partial \boldsymbol{\theta}^{\prime}\right)^{T}$, we write the following transformations:

$$
\left[\begin{array}{c}
\frac{\partial \lambda_{1 \|}}{\partial \boldsymbol{\theta}^{\prime}} \\
\frac{\partial \lambda_{1 \perp}}{\partial \boldsymbol{\theta}^{\prime}} \\
\frac{\partial \lambda_{2 \|}}{\partial \boldsymbol{\theta}^{\prime}} \\
\frac{\partial \lambda_{2 \perp}}{\partial \boldsymbol{\theta}^{\prime}}
\end{array}\right]=\left[\begin{array}{ccccc}
1 & \frac{1}{2} & 0 & \frac{1}{2} & 0 \cdots 0 \\
0 & 1 & 0 & 0 & 0 \cdots 0 \\
0 & \frac{1}{2} & 1 & \frac{1}{2} & 0 \cdots 0 \\
0 & 0 & 0 & 1 & 0 \cdots 0
\end{array}\right]
$$

based on the adopted parametrization described above in (A.25)

To compute $\mathrm{CRLB}_{\mathrm{FA}_{1,2}}=\left(\frac{\partial \mathrm{FA}_{1,2}}{\partial \boldsymbol{\theta}^{\prime}}\right) \mathbf{I}^{-1}\left(\frac{\partial \mathrm{FA}_{1,2}}{\partial \boldsymbol{\theta}^{\prime}}\right)^{T}$, the derivatives of $\mathrm{FA}_{1,2}$ to $\boldsymbol{\theta}^{\prime}$ are derived to be

$$
\left[\begin{array}{c}
\frac{\partial \mathrm{FA}_{1}}{\partial \boldsymbol{\theta}^{\prime}} \\
\frac{\partial \mathrm{FA} \mathrm{A}_{2}}{\partial \boldsymbol{\theta}^{\prime}}
\end{array}\right]=\left[\begin{array}{ccccc}
\frac{\partial \mathrm{FA} \mathrm{A}_{1}}{\partial \Delta \lambda_{1 \|}} & \frac{\partial \mathrm{FA}_{1}}{\partial \lambda_{1 \perp}} & 0 & \frac{\partial \mathrm{FA}_{1}}{\partial \lambda_{2} \perp} & 0 \cdots 0 \\
0 & \frac{\partial \mathrm{FA} A_{2}}{\partial \lambda_{1} \perp} & \frac{\partial F A_{2}}{\partial \Delta \lambda_{2} \|} & \frac{\partial \mathrm{FA} A_{2}}{\partial \lambda_{2} \perp} & 0 \cdots 0
\end{array}\right] .
$$

The derivatives of $\mathrm{FA}_{1}$ to the eigenvalues are

$$
\begin{aligned}
\frac{\partial \mathrm{FA}_{1}}{\partial \Delta \lambda_{1 \|}} & =4 \lambda_{1 \perp}\left(5 \lambda_{1 \perp}+2 \Delta \lambda_{1 \|}+\lambda_{2 \perp}\right) c_{1} \\
\frac{\partial \mathrm{FA}_{1}}{\partial \lambda_{1 \perp}} & =-2\left(\lambda_{2 \perp}+2 \Delta \lambda_{1 \|}\right)\left(5 \lambda_{1 \perp}+2 \Delta \lambda_{1 \|}+\lambda_{2 \perp}\right) c_{1} \\
\frac{\partial \mathrm{FA}_{1}}{\partial \lambda_{2 \perp}} & =2 \lambda_{1 \perp}\left(5 \lambda_{1 \perp}+2 \Delta \lambda_{1 \|}+\lambda_{2 \perp}\right) c_{1}
\end{aligned}
$$

with

$$
\begin{aligned}
c_{1}=\left(4 \Delta \lambda_{1 \|}^{2}+4 \Delta \lambda_{1 \|} \lambda_{1 \perp}\right. & +4 \Delta \lambda_{1 \|} \lambda_{2 \perp} \\
& \left.+9 \lambda_{1 \perp}^{2}+2 \lambda_{1 \perp} \lambda_{2 \perp}+\lambda_{2 \perp}^{2}\right)^{-3 / 2}
\end{aligned}
$$

while similarly for $\mathrm{FA}_{2}$

$$
\begin{aligned}
\frac{\partial \mathrm{FA}_{2}}{\partial \Delta \lambda_{2 \|}} & =4 \lambda_{2 \perp}\left(5 \lambda_{2 \perp}+2 \Delta \lambda_{2 \|}+\lambda_{1 \perp}\right) c_{1} \\
\frac{\partial \mathrm{FA}_{2}}{\partial \lambda_{1 \perp}} & =2 \lambda_{2 \perp}\left(5 \lambda_{2 \perp}+2 \Delta \lambda_{2 \|}+\lambda_{1 \perp}\right) c_{1} \\
\frac{\partial \mathrm{FA}_{2}}{\partial \lambda_{2 \perp}} & =-2\left(\lambda_{1 \perp}+2 \Delta \lambda_{2 \|}\right)\left(5 \lambda_{2 \perp}+2 \Delta \lambda_{2 \|}+\lambda_{1 \perp}\right) c_{1}
\end{aligned}
$$

with

$$
\begin{aligned}
c_{2}=\left(4 \Delta \lambda_{2 \|}^{2}+4 \Delta \lambda_{2 \|} \lambda_{1 \perp}\right. & +4 \Delta \lambda_{2 \|} \lambda_{2 \perp} \\
& \left.+\lambda_{1 \perp}^{2}+2 \lambda_{1 \perp} \lambda_{2 \perp}+9 \lambda_{2 \perp}^{2}\right)^{-3 / 2} .
\end{aligned}
$$




\section{ACKNOWLEDGMENT}

The authors would like to thank P. de Boer, M. Stam, T. Glatard, J. J. Keizer, J. Engelberts, and grid.support@ sara.nl for their contributions to this work. The authors would also like to thank Prof. I. T. Young for revising the manuscript.

\section{REFERENCES}

[1] P. Basser, S. Pajevic, C. Pierpaoli, J. Duda, and A. Aldroubi, "In vivo fiber tractography using DT-MRI data," Magn. Reson. Med., vol. 44, pp. 625-632, 2000.

[2] D. S. Tuch et al., "High angular resolution diffusion imaging of the human brain," in Proc. 8th ISMRM, Philadelphia, PA, 1999, p. 321.

[3] S.-K. Song, S.-W. Sun, W.-K. Ju, S.-J. Lin, A. H. Cross, and A. H. Neufeld, "Diffusion tensor imaging detects and differentiates axon and myelin degeneration in mouse optic nerve after retinal ischemia," $\mathrm{Neu}$ rolmage, vol. 20, pp. 1714-1722, 2003.

[4] M. D. Budde, M. Xie, A. H. Cross, and S. K. Song, "Axial diffusivity is the primary correlate of axonal injury in the experimental autoimmune encephalomyelitis spinal cord: A quantitative pixelwise analysis," $J$. Neurosci., vol. 29, pp. 2805-13, 2009.

[5] T. Hosey, G. Williams, and R. Ansorge, "Inference of multiple fiber orientations in high angular resolution diffusion imaging," Magn. Reson. Med., vol. 54, pp. 1480-89, 2005.

[6] M. Caan, K. Vermeer, L. van Vliet, C. Majoie, B. Peters, G. den Heeten, and F. Vos, "Shaving diffusion tensor images in discriminant analysis: A study into schizophrenia," Med. Image Anal., vol. 10, pp. 841-849, 2006.

[7] C. Sage, R. Peeters, A. Gorner, W. Robberecht, and S. Sunaert, "Quantitative diffusion tensor imaging in amyotrophic lateral sclerosis," $\mathrm{Neu}$ roimage, vol. 34, pp. 486-499, 2007.

[8] D. Alexander and G. Barker, "Optimal imaging parameters for fiberorientation estimation in diffusion MRI," Neuroimage, vol. 27, no. 2, pp. 357-367, 2005.

[9] L. R. Frank, "Anisotropy in high angular resolution diffusion-weighted MRI," Magn. Reson. Med., vol. 45, pp. 935-939, 2001.

[10] B. Kreher et al., "Multitensor approach for analysis and tracking of complex fiber configurations," Magn. Res. Med., vol. 54, pp. 1216-1225, 2005.

[11] S. Peled et al., "Geometrically constrained two-tensor model for crossing tracts in DWI," Magn Reson Med., vol. 24, pp. 1263-1270, 2006.

[12] E. Özarslan and T. H. Mareci, "Generalized diffusion tensor imaging and analytical relationships between diffusion tensor imaging and high angular resolution diffusion imaging," Magn. Reson. Med., vol. 50, pp. 955-965, 2003.

[13] J. D. Tournier, F. Calamante, and A. Connelly, "Robust determination of the fibre orientation distribution in diffusion MRI: Non-negativity constrained super-resolved spherical deconvolution," Neuroimage, vol. 35, no. 4, pp. 1459-1472, 2007.

[14] D. Tuch, "Diffusion MRI of complex tissue structure," Ph.D. dissertation, MIT, Cambridge, 2002.

[15] A. A. Qazi, A. Radmanesh, L. O’Donnell, G. Kindlmann, S. Peled, S. Whalen, C. F. Westin, and A. J. Golby, "Resolving crossings in the corticospinal tract by two-tensor streamline tractography: Method and clinical assessment using fMRI," Neuroimage, 2008.

[16] J. Malcolm, M. Shenton, and Y. Rathi, "Two-tensor tractography using a constrained filter," in Proc. Med. Image Computing Computer Assist. Intervent. (MICCAI), 2009, pp. 894-902.

[17] J. Malcolm, M. Shenton, and Y. Rathi, "Neural tractography using an unscented kalman filter," in Inf. Process. Med. Imag. (IPMI), 2009, pp. $126-138$.

[18] M. Descoteaux, R. Deriche, T. Knosche, and A. Anwander, "Deterministic and probabilistic tractography based on complex fibre orientation distributions," IEEE Trans. Med Imag., vol. 28, no. 2, pp. 269-286, Feb. 2009.

[19] A. J. den Dekker and J. Sijbers, "Estimation of signal and noise parameters from MR data," in Advanced Image Processing in Magnetic Resonance Imaging. Boca Raton, FL: CRC, 2005, vol. 27, Signal Process. Commun., ch. 4, pp. 85-143.

[20] J. L. Andersson, "Maximum a posteriori estimation of diffusion tensor parameters using a Rician noise model: Why, how and but," Neuroimage, vol. 42, pp. 1340-56, 2008.

[21] B. Landman, P. L. Bazin, and J. Prince, "Diffusion tensor estimation by maximizing Rician likelihood," in Proc. IEEE 11th ICCV, 2007, pp. 2433-40.
[22] P. Fillard, X. Pennec, V. Arsigny, and N. Ayache, "Clinical DT-MRI estimation, smoothing, and fiber tracking with log-euclidean metrics," IEEE Trans. Med. Imag., vol. 26, no. 11, pp. 1472-1482, Nov. 2007.

[23] S. Basu, T. Fletcher, and R. Whitaker, "Rician noise removal in diffusion tensor MRI,' in Proc. Miccai '06, 2006, vol. 4190, LNCS, pp. $117-125$.

[24] S. Aja-Fernandez, M. Niethammer, M. Kubicki, M. Shenton, and C.-F. Westin, "Restoration of DWI data using a Rician LMMSE estimator," IEEE Trans. Med. Imag., vol. 27, no. 10, pp. 1389-1403, Oct. 2008.

[25] C. G. Koay, L. C. Chang, C. Pierpaoli, and P. J. Basser, "Error propagation framework for diffusion tensor imaging via diffusion tensor representations," IEEE Trans. Med. Imag., vol. 26, no. 8, pp. 1017-1034, Aug. 2007.

[26] D. LeBihan, E. Breton, D. Lallemand, P. Grenier, E. Cabanis, and M. Laval-Jeantet, "MR imaging of intravoxel incoherent motions: Application to diffusion and perfusion in neurologic disorders," Radiology, vol. 161, pp. 401-407, 1986.

[27] C. Pierpaoli et al., "Diffusion tensor MR imaging of the human brain," Radiology, vol. 201, pp. 637-648, 1996.

[28] D. Jones, M. Horsfield, and A. Simmons, "Optimal strategies for measuring diffusion in anisotropic systems by Magnetic Resonance Imaging," Magn. Reson. Med., vol. 42, pp. 515-525, 1999.

[29] Y. Bito, S. Hirata, and E. Yamamoto, "Optimal gradient factors for ADC measurements," in Proc. ISMRM, 1995, p. 913.

[30] S. Peled, "New perspectives on the sources of white matter DTI signal," IEEE Trans. Med. Imag., vol. 26, no. 11, pp. 1448-55, Nov. 2007.

[31] Y. Assaf and P. J. Basser, "Composite hindered and restricted model of diffusion (CHARMED) MR imaging of the human brain," $\mathrm{Neu}$ roImage, vol. 27, pp. 48-58, 2005.

[32] P. Kingsley and W. Monahan, "Selection of the optimum b-factor for diffusion-weighted magnetic resonance imaging assessment of ischemic stroke," Magn. Reson. Med., vol. 51, pp. 996-1001, 2004.

[33] T. Behrens, H. Berg, S. Jbabdi, M. Rushworth, and M. Woolricha, "Probabilistic diffusion tractography with multiple fibre orientations: What can we gain?," NeuroImage, vol. 34, pp. 144-155, 2007.

[34] Y. Assaf, D. Ben-Bashat, J. Chapman, S. Peled, I. Biton, M. Kafri, Y. Segev, T. Hendler, A. Korczyn, M. Graif, and Y. Cohen, "High b-value q-space analyzed diffusion-weighted MRI: Application to Multiple Sclerosis," Magn. Reson. Med., vol. 47, pp. 115-126, 2002.

[35] D. K. Jones and P. J. Basser, "Squashing peanuts and smashing pumpkins: How noise distorts diffusion-weighted MR data," Magn. Reson. Med., vol. 52, no. 5, pp. 979-993, 2004.

[36] O. Brihuega-Moreno, F. P. Heese, and L. D. Hall, "Optimization of diffusion measurements using cramer-rao lower bound theory and its application to articular cartilage," Magn. Reson. Med., vol. 50, no. 5, pp. 1069-76, 2003.

[37] D. C. Alexander, "A general framework for experiment design in diffusion MRI and its application in measuring direct tissue-microstructure features," Magn. Reson. Med., vol. 60, no. 2, pp. 439-48, 2008.

[38] P. Basser and C. Pierpaoli, "Microstructural and physiological features of tissues elucidated by quantitative-diffusion-tensor MRI," J. Magn. Reson. B, vol. 111, pp. 209-219, 1996.

[39] H. Gudbjartsson and S. Patz, "The Rician distribution of noise MRI data," Magn. Res. Red., pp. 910-914, 1995.

[40] A. van den Bos, Parameter Estimation for Scientists and Engineers Hoboken, NJ: Wiley., 2007.

[41] A. J. den Dekker, S. van Aert, A. van den Bos, and D. van Dyck, "Maximum likelihood estimation ofstructure parameters from high resolution electron microscopy images. Part I: A theoretical framework," $U l$ tramicroscopy, vol. 104, pp. 83-106, 2005.

[42] J. Sijbers and A. J. den Dekker, "Maximum likelihood estimation of signal amplitude and noise variance from MR data," Magn. Reson. Med., vol. 51, pp. 586-594, 2004.

[43] O. T. Karlsen, R. Verhagen, and W. Bovee, "Parameter estimation from Rician-distributed data sets using a maximum likelihood estimator: Application to T-1 and perfusion measurements," Magn. Reson. Med., vol. 41, no. 3, pp. 614-623, 1999.

[44] C. Pierpaoli and D. K. Jones, "Removing CSF contamination in brain DT-MRIs by using a two-compartment tensor model," in Proc. ISMRM, 2005, p. 1215.

[45] J. D. Carew, C. G. Koay, G. Wahba, A. L. Alexander, M. E. Meyerand, and P. J. Basser, The asymptotic behavior of the nonlinear estimators of the diffusion tensor and tensor-derived quantities with implications for group analysis Univ. Wisconsin, Tech. Rep. 1132, 2006, Tech. Rep. 1132 . 
[46] J. D. Tournier, C. H. Yeh, F. Calamante, K. H. Cho, A. Connelly, and C. P. Lin, "Resolving crossing fibres using constrained spherical deconvolution: Validation using diffusion-weighted imaging phantom data," Neuroimage, vol. 42, no. 2, pp. 617-625, 2008.

[47] J.-F. Mangin, C. Poupon, C. Clark, D. L. Bihan, and I. Bloch, "Eddycurrent distortion correction and robust tensor estimation for MR diffusion imaging," in Proc. MICCAI'01, 2006, pp. 186-193.

[48] M. Caan et al., "Adaptive noise filtering for accurate and precise diffusion estimation in fiber crossings," in Proc. MICCAI, 2010.

[49] S. Olabarriaga, T. Glatard, K. Boulebiar, and P. T. de Boer, "From 'low-hanging' to 'user-ready': Initial steps into a healthgrid," in HealthGrid. Chicago, IL: IOS Press, 2008, Studies Health Technol. Informatics, pp. 70-79.

[50] N. Toussaint, A. van Muiswinkel, F. G. Hoogenraad, R. Holthuizen, and S. Sunaert, "Resolving fiber crossings: A two fiber model simulation result," in Proc. ISMRM, 2005, vol. 13, p. 1339.
[51] V. Prckovska, A. F. Roebroeck, W. Pullens, A. Vilanova, and B. M. T. Romeny, "Optimal acquisition schemes in high angular resolution diffusion weighted imaging," in Proc. MICCAI, 2008, vol. 5242, pp. $9-17$.

[52] O. Pasternak, Y. Assaf, N. Intrator, and N. Sochen, "Variational multiple-tensor fitting of fiber-ambiguous diffusion-weighted magnetic resonance imaging voxels," Magn. Reson. Imag., vol. 26, pp. 1133-44, 2008.

[53] H. E. Assemlal, D. Tschumperle, and L. Brun, "Efficient and robust computation of PDF features from diffusion $\mathrm{mr}$ signal," Med. Image Anal., vol. 13, no. 5, pp. 715-29, 2009.

[54] T. P. Hosey, S. G. Harding, T. A. Carpenter, R. E. Ansorge, and G. B Williams, "Application of a probabilistic double-fibre structure model to diffusion-weighted MR images of the human brain," Magn. Reson. Imag., vol. 26, pp. 236-45, 2008. 